\title{
Radial velocities and metallicities from infrared Ca II triplet spectroscopy of open clusters
}

\section{Berkeley 26, Berkeley 70, NGC 1798, and NGC 2266^}

\author{
R. Carrera
}

\author{
Instituto de Astrofísica de Canarias, 38203 La Laguna, Tenerife, Spain \\ Departamento de Astrofísica, Universidad de La Laguna, 38207 La Laguna, Tenerife, Spain \\ e-mail: rcarrera@iac.es
}

Received 18 May 2012 / Accepted 8 July 2012

\begin{abstract}
Context. Open clusters are ideal test particles for studying the formation and evolution of the Galactic disk. However, the number of clusters with information about their radial velocities and chemical compositions remains largely insufficient.

Aims. We attempt to increase the number of open clusters with determinations of radial velocities and metallicities from spectroscopy. Methods. We acquired medium-resolution spectra $(R \sim 8000)$ in the region of the infrared Ca II triplet lines $(\sim 8500 \AA)$ for several stars in four open clusters with the long-slit spectrograph IDS at the $2.5 \mathrm{~m}$ Isaac Newton Telescope, Roque de los Muchachos Observatory, Spain. Radial velocities were obtained by cross-correlating the observed spectra with those of two template stars. We used the relationships available in the literature between the strength of infrared Ca II lines and metallicities to derive the metal content of each cluster.

Results. We provide the first spectroscopic determinations of radial velocities and metallicities for the open clusters Berkeley 26 , Berkeley 70, NGC 1798, and NGC 2266. We obtain $\left\langle V_{r}\right\rangle=68 \pm 12,-15 \pm 7,2 \pm 10$, and $-16 \pm 15 \mathrm{~km} \mathrm{~s}^{-1}$ for Berkeley 26, Berkeley 70 , NGC 1798, and NGC 2266, respectively. For Berkeley 26 we derive a metallicity of $[\mathrm{Fe} / \mathrm{H}]=-0.35 \pm 0.17$ dex. Berkeley 70 has a solar metallicity of $[\mathrm{Fe} / \mathrm{H}]=-0.01 \pm 0.14 \mathrm{dex}$, while NGC 1798 has a slightly lower metal content of $[\mathrm{Fe} / \mathrm{H}]=-0.12 \pm 0.07 \mathrm{dex}$. Finally, we derive a metallicity of $[\mathrm{Fe} / \mathrm{H}]=-0.38 \pm 0.06$ dex for NGC 2266.
\end{abstract}

Key words. open clusters and associations: individual: Berkeley 26 - open clusters and associations: individual: Berkeley 70 open clusters and associations: individual: NGC 1798 - open clusters and associations: individual: NGC 2266 - stars: abundances Galaxy: disk

\section{Introduction}

Open clusters (OCs) have been widely used to investigate the existence of trends in the Galactic disk such as radial and vertical metallicity gradients or an age-metallicity relationship (e.g. Janes 1979; Panagia \& Tosi 1980; Twarog et al. 1997; Friel et al. 2002; Chen et al. 2003; Pancino et al. 2010; Carrera \& Pancino 2011). However, our knowledge of OC properties is still far from complete. Age and distance estimations, mainly obtained from isochrone fitting, are available for about $\sim 70 \%$ of the more than 2100 OCs known in our Galaxy (Dias et al. 2002) ${ }^{1}$. However, radial velocities have been determined only for $24 \%$ of them. The picture is even worse in the case of chemical compositions, which have been obtained for only $9 \%$, mainly by means of different studies in the Washington (e.g. Parisi et al. 2005), DDO (e.g. Janes 1977; Clariá et al. 1999), Strömgren (e.g. Paunzen et al. 2003), UBV (e.g. Clariá et al. 1998), IR (e.g. Carraro et al. 1999; Vallenari et al. 2000), and Vilnius (e.g. Bartašiūtė et al. 2011; Zdanavičius et al. 2011) photometric systems, often giving

\footnotetext{
* Based on observations made with the 2.5 Isaac Newton Telescope operated on the island of La Palma by the Isaac Newton Group in the Spanish Observatorio del Roque de los Muchachos of the Instituto de Astrofísica de Canarias.

1 The updated version of this catalog can be found at http://www. astro.iag.usp.br/ wilton/
}

rise to considerable differences among them and to those obtained from spectroscopy.

Reliable information about the chemical composition can be retrieved only from spectroscopy. However, the acquisition of high-resolution spectra $(R \geq 20000)$, which is the only way to derive detailed abundances, needs large amounts of telescope time. This together with the complexity of the associated analysis explain why this kind of study has been performed for only $4 \%$ of known OCs (Carrera \& Pancino 2011). The alternative is to perform low- and medium-resolution spectroscopy, although these data provide information only about the metallicities and radial velocities of stars (e.g. Friel \& Janes 1993; Friel et al. 2002; Carrera et al. 2007; Warren \& Cole 2009).

The goal of this paper is to increase the number of clusters with radial velocities and metallicities determined from spectroscopy. For this purpose, we have selected four OCs, Berkeley 26, Berkeley 70, NGC 1798, and NGC 2266, that had not been studied spectroscopically before. These clusters are located towards the Galactic anticenter, at relatively large distances $\left(R_{\mathrm{GC}} \geq 10 \mathrm{kpc}\right)$ where the trend of the radial metallicity seems to flatten (e.g. Carrera \& Pancino 2011). Medium-resolution spectra in the region of the near-infrared $\mathrm{Ca}$ II triplet $(\mathrm{CaT})$ at $\sim 8500 \AA$ were obtained for several red giant branch (RGB) stars in each cluster. The observations and data reduction are described in Sect. 2, radial velocities and metallicities are obtained 


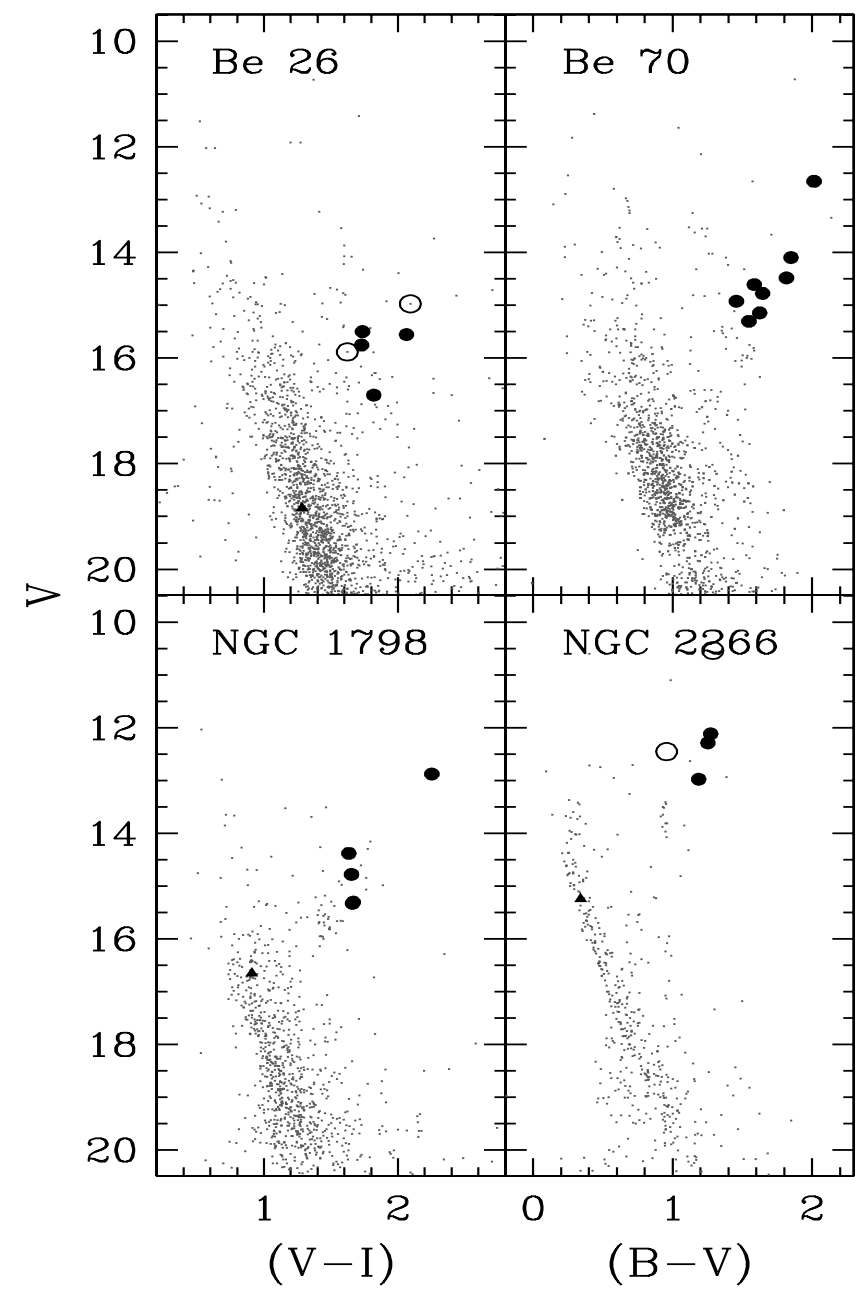

Fig. 1. Color-magnitude diagrams of clusters studied in this paper (small gray dots). Filled circles are stars assumed to be cluster members based on their radial velocities and used in the metallicity determination. Filled triangles are main-sequence stars assumed to be members based on their radial velocity but not used to determine the metallicity of these clusters. Open circles are stars rejected as possible members based on their radial velocities.

in Sects. 3, 4, the results for each cluster are discussed and compared with the literature in Sect. 5, and the comparison of the results obtained here with the trends observed in the disk is performed in Sect. 6. Finally, our main conclusions are summarised in Sect. 7.

\section{Observations and data reduction}

The target stars were selected from the color-magnitude diagram of each cluster taken from Piatti et al. (2010), Ann et al. (2002), Park \& Lee (1999), and Maciejewski \& Niedzielski (2007) for Berkeley 26, Berkeley 70, NGC 1798, and NGC 2266 , respectively (Fig. 1). These data together with the coordinates of each target stars were obtained from the WEBDA ${ }^{2}$ database (Mermilliod 1995). In total, we observed 26 stars: 8 in Berkeley 70, and 6 in the others. Table 1 summarizes the coordinates, magnitudes, exposure times, and signal-to-noise ratios for each target star. The global properties of each cluster are listed in Table 2.

\footnotetext{
${ }^{2}$ http://www. univie.ac.at/webda
}

Observations were carried out between the nights of 5 and 9 of February 2012 using the Intermediate Dispersion Spectrograph (IDS) mounted at the Cassegrain focus of the $2.5 \mathrm{~m}$ Isaac Newton Telescope (INT) located at the Roque de los Muchachos Observatory, Spain. We used the R1200R grism centered at $8500 \AA$ and the RED +2 CCD, providing a spectral resolution of about 8000 . For each target, we obtained two exposures with the star shifted along the slit in each of them. In some cases, another star was observed together with the main target because it was aligned with the slit. Since stellar spectra have reasonable signal-to-noise ratios, we also analyzed these stars. In all cases, these are main-sequence stars, which therefore, were used only for the radial velocity analysis.

The data reduction was performed using imred and specred packages within IRAF ${ }^{3}$. Firstly, each image was overscansubtracted and flat-field corrected. Then, since we had acquired two images of each target with the star shifted along the slit, we subtracted one from the other, obtaining a positive and a negative spectrum in the same image. With this procedure, the sky was subtracted from the same physical pixel in which the star was observed, thus minimizing the effects of pixel-to-pixel sensitivity variations. A time dependence of course remained, since the two spectra had not been taken simultaneously. These sky residuals were eliminated in the following step, in which the spectrum was extracted in the traditional way and the remaining sky background subtracted from the information on both sides of the stellar spectra. In the next step, the spectrum was wavelengthcalibrated. We then again subtracted the negative from the positive spectrum (so we effectively added both spectra because one is negative) to obtain the final spectrum. Finally, each spectrum was normalized by fitting a polynomial, excluding the strongest lines in the wavelength range, such as those of the CaT.

\section{Radial velocities}

The radial velocity of each star was calculated using the fxcor task in IRAF, which performs a cross-correlation between the target and template spectra of known radial velocity. As templates, we used two bright stars, HD 15656 and HD 74462, observed in the same nights and with a high signal-to-noise ratio. The final radial velocity for each target star was obtained as the average of the velocities obtained for each template, weighted by the width of the correlation peaks. The measured radial velocities for each star are listed in Col. 8 of Table 1 . The velocity distribution of each cluster is shown in Fig. 2 and discussed in Sect. 5.

\section{CaT equivalent widths and metallicity determination}

The equivalent widths of the $\mathrm{CaT}$ lines of the observed RGB stars were used to determine the metallicity of each cluster. This technique was originally developed to determine the metallicities of old and metal-poor populations such as those of globular clusters (e.g. Armandroff \& Zinn 1988; Armandroff \& Da Costa 1991; Rutledge et al. 1997). However, more recent investigations have extended its validity to much larger ranges of ages and metallicities, including extremely metal-poor regimes (Starkenburg et al. 2010) and the young and metal-rich OC populations (Cole et al. 2004; Carrera et al. 2007).

\footnotetext{
${ }^{3}$ Image Reduction and Analysis Facility, IRAF is distributed by the National Optical Astronomy Observatories, which are operated by the Association of Universities for Research in Astronomy, Inc., under cooperative agreement with the National Science Foundation.
} 
Table 1. Observing logs and program star information.

\begin{tabular}{|c|c|c|c|c|c|c|c|c|c|c|c|c|c|c|}
\hline Cluster & Star* & $\begin{array}{c}\alpha_{2000} \\
(\mathrm{~h})\end{array}$ & $\begin{array}{l}\delta_{2000} \\
(\mathrm{deg})\end{array}$ & $\begin{array}{c}V \\
(\mathrm{mag})\end{array}$ & $\begin{array}{r}V-I \\
(\mathrm{mag})\end{array}$ & $\begin{array}{l}B-V \\
(\mathrm{mag})\end{array}$ & $\begin{array}{c}V_{r} \\
\left(\mathrm{~km} \mathrm{~s}^{-1}\right) \\
\end{array}$ & $\begin{array}{c}E W_{8498} \\
(\AA)\end{array}$ & $\begin{array}{c}E W_{8542} \\
(\AA)\end{array}$ & $\begin{array}{c}E W_{8662} \\
(\AA)\end{array}$ & $\begin{array}{c}\Sigma \mathrm{Ca} \\
(\AA)\end{array}$ & $\begin{array}{c}t_{\exp } \\
(\mathrm{sec})\end{array}$ & $S / N^{\text {tot }}$ & Note \\
\hline \multirow[t]{6}{*}{ Be 26} & 1038 & $06: 50: 02.9$ & $+05: 44: 59$ & 18.845 & 1.283 & - & $66.1 \pm 11.9$ & - & - & - & - & $2 \times 950$ & 25 & 2 \\
\hline & 1155 & 06:50:09.5 & $+05: 44: 28$ & 15.754 & 1.729 & - & $71.0 \pm 7.9$ & $1.17 \pm 0.13$ & $3.39 \pm 0.12$ & $2.74 \pm 0.12$ & $7.30 \pm 0.21$ & $2 \times 950$ & 29 & 1 \\
\hline & 1231 & 06:50:08.6 & $+05: 44: 07$ & 16.702 & 1.819 & - & $66.1 \pm 7.7$ & $1.13 \pm 0.14$ & $3.21 \pm 0.21$ & $2.77 \pm 0.10$ & $7.11 \pm 0.27$ & $2 \times 1500$ & 25 & 1 \\
\hline & 1288 & 06:50:05.7 & $+05: 43: 53$ & 15.500 & 1.735 & - & $68.3 \pm 7.2$ & $1.27 \pm 0.11$ & $3.51 \pm 0.12$ & $2.93 \pm 0.12$ & $7.71 \pm 0.20$ & $2 \times 850$ & 29 & 1 \\
\hline & 1421 & $06: 50: 18.4$ & $+05: 43: 22$ & 15.556 & 2.063 & - & $68.3 \pm 6.7$ & $1.45 \pm 0.08$ & $3.94 \pm 0.09$ & $2.62 \pm 0.07$ & $8.01 \pm 0.14$ & $2 \times 900$ & 40 & 1 \\
\hline & 1650 & $06: 50: 14.9$ & $+05: 42: 17$ & 14.974 & 2.092 & - & $97.1 \pm 7.8$ & - & - & - & - & $2 \times 850$ & 45 & 9 \\
\hline \multirow[t]{8}{*}{ Be 70} & 1105 & $05: 25: 44.8$ & $+41: 56: 44$ & 12.651 & 2.035 & 2.014 & $-13.8 \pm 7.6$ & $1.94 \pm 0.05$ & $4.66 \pm 0.05$ & $3.50 \pm 0.06$ & $10.10 \pm 0.09$ & $2 \times 700$ & 186 & 1 \\
\hline & 1088 & $05: 25: 45.0$ & $+41: 55: 55$ & 14.097 & 1.994 & 1.849 & $-4.3 \pm 7.5$ & $1.61 \pm 0.02$ & $4.32 \pm 0.02$ & $3.20 \pm 0.03$ & $9.13 \pm 0.04$ & $2 \times 800$ & 98 & 1 \\
\hline & 0820 & $05: 25: 49.9$ & $+41: 56: 51$ & 14.482 & 1.944 & 1.816 & $-17.3 \pm 7.9$ & $1.53 \pm 0.04$ & $4.18 \pm 0.03$ & $2.99 \pm 0.04$ & $8.70 \pm 0.06$ & $2 \times 800$ & 69 & 1 \\
\hline & 0609 & $05: 25: 54.6$ & $+41: 58: 22$ & 14.608 & 1.734 & 1.585 & $-11.7 \pm 7.2$ & $1.56 \pm 0.04$ & $3.69 \pm 0.05$ & $2.80 \pm 0.06$ & $8.05 \pm 0.09$ & $2 \times 850$ & 44 & 1 \\
\hline & 0811 & $05: 25: 50.1$ & $+41: 57: 46$ & 14.774 & 1.796 & 1.645 & $-19.7 \pm 7.7$ & $1.46 \pm 0.08$ & $3.87 \pm 0.07$ & $2.94 \pm 0.08$ & $8.27 \pm 0.12$ & $2 \times 850$ & 58 & 1 \\
\hline & 0690 & $05: 25: 52.7$ & $+41: 57: 13$ & 14.923 & 1.617 & 1.457 & $-20.4 \pm 7.3$ & $1.49 \pm 0.05$ & $3.71 \pm 0.07$ & $2.71 \pm 0.06$ & $7.91 \pm 0.11$ & $2 \times 950$ & 58 & 1 \\
\hline & 0735 & $05: 25: 51.9$ & $+41: 57: 35$ & 15.142 & 1.762 & 1.624 & $-19.7 \pm 7.9$ & $1.46 \pm 0.08$ & $3.89 \pm 0.08$ & $2.80 \pm 0.10$ & $8.15 \pm$ & & 0 & 1 \\
\hline & & $05: 26: 03.3$ & $+41: 58: 54$ & 15.304 & 1.716 & 1.547 & $-12.9 \pm 8.9$ & $1.35 \pm 0.12$ & $4.01 \pm 0.13$ & $2.35 \pm 0.13$ & $7.71 \pm 0.22$ & $2 \times 900$ & 30 & 1 \\
\hline \multirow[t]{6}{*}{ NGC 1798} & 0005 & $05: 11: 36.7$ & +474149 & 14.380 & 1.633 & 1.483 & $4.3 \pm 7.3$ & $1.54 \pm 0.06$ & $3.79 \pm 0.07$ & $2.91 \pm 0.07$ & $8.24 \pm 0.12$ & $2 \times 800$ & 56 & 1 \\
\hline & 0009 & $05: 11: 42.8$ & $+47: 41: 32$ & 14.777 & 1.653 & 1.486 & $0.2 \pm 7.3$ & $1.31 \pm 0.10$ & $3.45 \pm 0.10$ & $2.60 \pm 0.08$ & $7.36 \pm 0.16$ & $2 \times 750$ & 40 & 1 \\
\hline & 0011 & 05:11:37.0 & $+47: 40: 15$ & 15.305 & 1.666 & 1.529 & $-3.4 \pm 7.0$ & $1.40 \pm 0.09$ & $3.85 \pm 0.11$ & $2.71 \pm 0.08$ & $7.96 \pm 0.16$ & $2 \times 800$ & 41 & 1 \\
\hline & 0013 & $05: 11: 41.2$ & $+47: 40: 41$ & 15.325 & 1.660 & 1.524 & $-5.6 \pm 7.2$ & $1.38 \pm 0.07$ & $3.52 \pm 0.10$ & $2.64 \pm 0.13$ & $7.54 \pm 0.18$ & $2 \times 850$ & 40 & 1 \\
\hline & 0043 & $05: 11: 47.9$ & $+47: 40: 26$ & 12.875 & 2.252 & 1.970 & $11.2 \pm 7.8$ & $1.89 \pm 0.02$ & $4.68 \pm 0.03$ & $3.44 \pm 0.03$ & $10.01 \pm 0.05$ & $2 \times 700$ & 110 & 1 \\
\hline & 0608 & 05:11:41.0 & $+47: 40: 43$ & 16.653 & 0.909 & 0.816 & $12.8 \pm 11.0$ & - & - & - & - & $2 \times 850$ & 25 & 2 \\
\hline \multirow[t]{6}{*}{ NGC 2266} & 0034 & $06: 43: 16.0$ & +265745 & 12.454 & - & 0.957 & $21.0 \pm 7.7$ & - & - & - & - & $2 \times 350$ & 55 & 9 \\
\hline & 0067 & $06: 43: 20.2$ & $+26: 57: 32$ & 10.538 & - & 1.288 & $30.2 \pm 7.2$ & - & - & - & - & $2 \times 50$ & 55 & 9 \\
\hline & 0075 & $06: 43: 12.9$ & $+26: 57: 07$ & 15.242 & - & 0.341 & $-18.0 \pm 9.2$ & - & - & - & - & $2 \times 350$ & 24 & 2 \\
\hline & 0096 & $06: 43: 28.0$ & $+26: 58: 10$ & 12.287 & - & 1.253 & $-8.9 \pm 7.5$ & $1.47 \pm 0.05$ & $3.84 \pm 0.05$ & $2.65 \pm 0.06$ & $7.96 \pm 0.09$ & $2 \times 250$ & 58 & 1 \\
\hline & 0101 & $06: 43: 25.3$ & $+26: 57: 49$ & 12.978 & - & 1.187 & $-19.1 \pm 7.8$ & $1.46 \pm 0.06$ & $3.71 \pm 0.06$ & $2.25 \pm 0.10$ & $7.42 \pm 0.13$ & $2 \times 500$ & 50 & 1 \\
\hline & 1011 & $06: 43: 25.3$ & $+26: 57: 50$ & 12.117 & - & 1.273 & $-18.5 \pm 7.1$ & $1.43 \pm 0.06$ & $4.13 \pm 0.05$ & $2.97 \pm 0.06$ & $8.53 \pm 0.10$ & $2 \times 500$ & 58 & 1 \\
\hline
\end{tabular}

Notes. ${ }^{(*)}$ Identification taken from WEBDA database. (1) Member star used both for radial velocity and metallicity determination; (2) member star used only for radial velocity determination; (9) non-member star.

Table 2. Adopted cluster parameters.

\begin{tabular}{lccccc}
\hline \hline Cluster & $\begin{array}{c}E(B-V) \\
(\mathrm{mag})\end{array}$ & $\begin{array}{c}(m-M)_{0} \\
(\mathrm{mag})\end{array}$ & $\begin{array}{c}\text { Age } \\
(\mathrm{Gyr})\end{array}$ & $\begin{array}{c}R_{\mathrm{gc}} \\
(\mathrm{kpc})\end{array}$ & $\begin{array}{c}z \\
(\mathrm{kpc})\end{array}$ \\
\hline Be 26 & $0.68 \pm 0.06$ & $13.8 \pm 0.5$ & $4.25 \pm 0.25$ & 13.9 & 0.24 \\
$\mathrm{Be} 70^{b}$ & $0.48 \pm 0.10$ & $13.1 \pm 0.3$ & $4.0 \pm 0.8$ & 12.6 & 0.26 \\
${\text { NGC } 1798^{c}}^{c}$ & $0.51 \pm 0.04$ & $13.1 \pm 0.2$ & $1.4 \pm 0.3$ & 12.5 & 0.35 \\
NGC 2266 $^{d}$ & $0.15 \pm 0.01$ & $12.6 \pm 0.2$ & $0.7 \pm 0.1$ & 11.8 & 0.59 \\
\hline
\end{tabular}

Notes. ${ }^{(a)}$ Average of the values by Piatti et al. (2010) and Hasegawa et al. (2008). See text for details. ${ }^{(b)}$ Values taken from Ann et al. (2002) which are similar to those derived by Hasegawa et al. (2004) with the exception that they derived an age of $2.8 \mathrm{Gyr} .{ }^{(c)}$ Values taken from Park \& Lee (1999) which are similar to others available in the literature within error bars (e.g. Dutra \& Bica 2000; Tadross et al. 2002; Salaris et al. 2004; Maciejewski \& Niedzielski 2007). ${ }^{(d)}$ Values taken from Dias et al. (2012). Similar values have been obtained by other investigations in this cluster (Kaluzny \& Mazur 1991; Dutra \& Bica 2000; Tadross 2001; Tadross et al. 2002; Lata et al. 2002; Salaris et al. 2004; Paunzen \& Netopil 2006; Maciejewski \& Niedzielski 2007; Maciejewski et al. 2008; Paunzen et al. 2010; Monteiro et al. 2010).

Although the procedure used to determine metallicities from the CaT lines strengths is described in depth by Carrera et al. (2007), we summarize here the main steps. Firstly, we determine the equivalent width of each line by fitting its profile with a Gaussian plus a Lorentzian. This combination provides the best fit to the line core and wings. Although in our case the spectra had already been normalized, the position of the continuum was recalculated by performing a linear fitting to the mean values of each continuum bandpass. The bandpasses used to fit the line
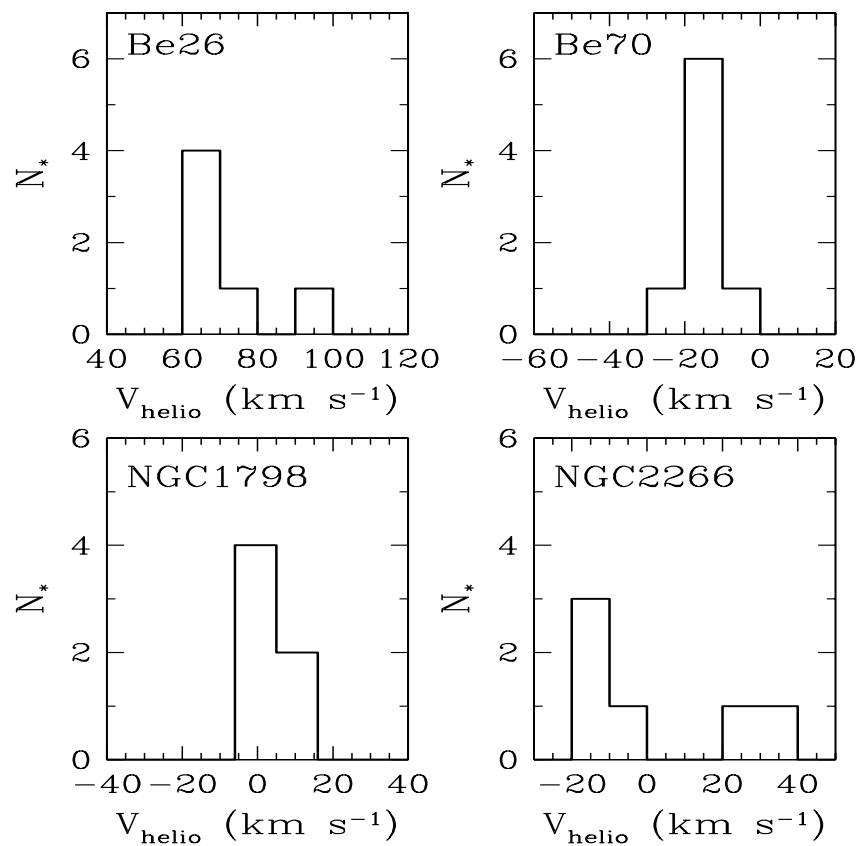

Fig. 2. Radial velocity distributions of the four clusters studied.

profile and determine the continuum position are those described by Cenarro et al. (2001). The CaT index, denoted $\Sigma \mathrm{Ca}$, is defined as the sum of the equivalent widths of the three CaT lines. We list the equivalent widths of the three CaT lines and the $\Sigma \mathrm{Ca}$ values obtained for each target star in Table 1. 
Table 3. Cluster metallicities and radial velocities.

\begin{tabular}{lccccc}
\hline \hline Cluster & $\begin{array}{c}W_{V}^{\prime} \\
(\mathrm{mag})\end{array}$ & $\begin{array}{c}{[\mathrm{Fe} / \mathrm{H}]_{V}} \\
(\mathrm{dex})\end{array}$ & $\begin{array}{c}W_{I}^{\prime} \\
(\mathrm{mag})\end{array}$ & $\begin{array}{c}{[\mathrm{Fe} / \mathrm{H}]_{I}} \\
(\mathrm{dex})\end{array}$ & $\begin{array}{c}\left\langle V_{r}\right\rangle \\
\left(\mathrm{km} \mathrm{s}^{-1}\right)\end{array}$ \\
\hline Be 26 & $7.42 \pm 0.20$ & $-0.43 \pm 0.20$ & $6.88 \pm 0.23$ & $-0.35 \pm 0.17$ & $68 \pm 12$ \\
Be 70 & $8.37 \pm 0.01$ & $-0.02 \pm 0.18$ & $7.67 \pm 0.01$ & $-0.01 \pm 0.14$ & $-15 \pm 7$ \\
NGC 1798 & $8.05 \pm 0.04$ & $-0.16 \pm 0.10$ & $7.41 \pm 0.03$ & $-0.12 \pm 0.07$ & $2 \pm 10$ \\
NGC 2266 & $7.54 \pm 0.02$ & $-0.38 \pm 0.07$ & - & - & $-16 \pm 15$ \\
\hline
\end{tabular}

The strengths of the CaT lines do not only depend on the chemical abundance, but also on the temperature and gravity of the stellar atmosphere. Since we are only interested in the abundance dependence, the temperature and gravity dependences must be removed. To do this, we used the finding that, for a given chemical abundance, the stars define a sequence in the luminosity- $\Sigma \mathrm{Ca}$ plane when the temperature and/or gravity change. It is known that this sequence is not linear for extremely metal-poor regimes (Starkenburg et al. 2010) and in the bottom of the RGB (Carrera et al. 2007). However, we can neglect this trend since our targets are located in the upper part of the RGB and because for OCs we expect metallicities of around solar. Traditionally, the difference between the $V$ magnitude of a star and that of the horizontal branch (HB), $V-V_{\mathrm{HB}}$, has been used as a luminosity indicator, which also removes any dependence on distance and reddening (e.g. Armandroff \& Da Costa 1991; Rutledge et al. 1997; Cole et al. 2004). However, in many stellar populations, it is difficult to define accurately the position of the $\mathrm{HB}$, such as poorly populated OCs. For this reason, other authors have used the absolute magnitude in either the $V$ or $I$ bandpasses (e.g. Armandroff \& Da Costa 1991; Pont et al. 2004; Carrera et al. 2007). We used this second approach in this paper. The CaT metallicity calibration based on $M_{I}$ is less sensitive to age. Unfortunately, I magnitudes are unavailable for the stars of one of the clusters, NGC 2266. Therefore, we derive the metallicities of each cluster using both $M_{V}$ and $M_{I}$ to minimize the effect of age on the metallicity determination of NGC 2266.

In the $M_{V, I}-\Sigma \mathrm{Ca}$ planes, the sequences can be parametrized in the form $\Sigma \mathrm{Ca}=W_{V, I}^{\prime}+\beta_{V, I} \times M_{V, I}$. The slopes $\beta_{V, I}$ are independent of metallicity as demonstrated by Carrera et al. (2007), whereas $W_{V, I}^{\prime}$, the so-called reduced equivalent width, varies as a function of the chemical abundance. We used the slopes $\beta_{V}=-0.677 \pm 0.004 \AA \mathrm{mag}^{-1}$ and $\beta_{I}=-0.611 \pm 0.002 \AA \mathrm{mag}^{-1}$ calculated by Carrera et al. (2007). The position of the observed stars in each cluster in the $M_{V}-\Sigma \mathrm{Ca}$ and $M_{I}-\Sigma \mathrm{Ca}$ planes are shown in Figs. 3 and 4, respectively. The $W_{V, I}^{\prime}$ values obtained for each cluster are listed in Table 3.

Relationships between $W_{V, I}^{\prime}$ and metallicity on different metallicity scales, including the widely used one from Carretta \& Gratton (1997) were derived by Carrera et al. (2007). Carretta et al. (2009) updated their metallicity scale based on more homogeneous and higher resolution spectra of a larger sample of bright giants in 24 Galactic globular clusters. They provided a detailed comparison between their old and new scales. The existence of this new metallicity scale for globular clusters and the new determinations of metallicities available for some open clusters used by Carrera et al. (2007) to derive their calibrations, motivated Carrera et al. (2011) to obtain new relationships between $W_{V, I}^{\prime}$ and metallicity. We used these updated relations to obtain the metallicity, on the Carretta et al. (2009) scale, of the clusters studied in this paper. The values obtained for each cluster are listed in Table 3 and discussed below. We have to note that the metallicities derived are related to the reddenings and distance moduli used as references in each case. For this reason,
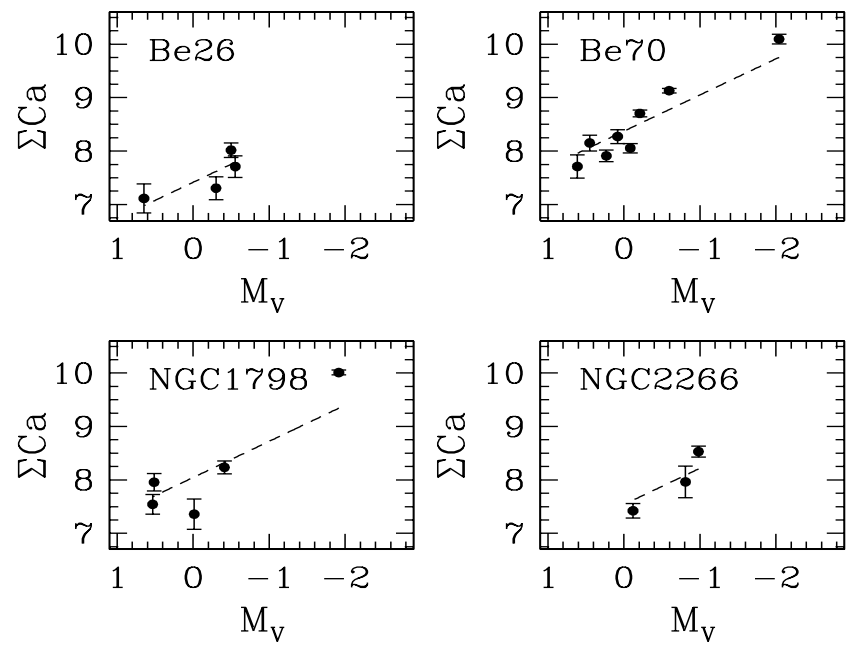

Fig. 3. Observed stars in each cluster in the $M_{V}-\Sigma C$ a plane. Dashed lines are the linear fit to the stars in each cluster.
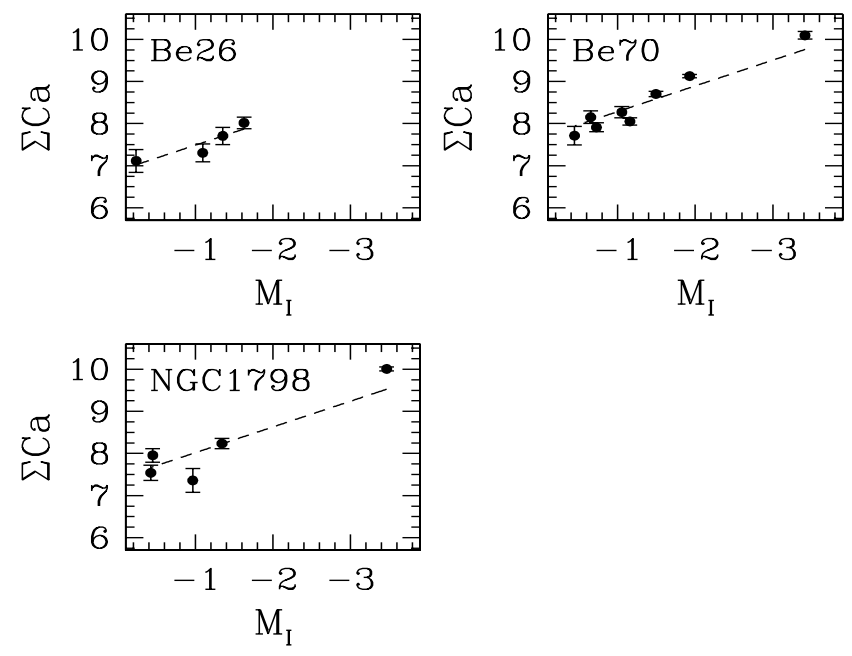

Fig. 4. Same as Fig. 3 but for $M_{I}-\Sigma \mathrm{Ca}$ plane.

the metallicity error bars were estimated by taken into account the uncertainties in these quantities.

\section{Cluster-by-cluster discussion}

\subsection{Berkeley 26}

Also known as Biurakan 12, Berkeley 26 is a poorly studied cluster in Monoceros. To our knowledge, there are only three studies of this cluster in the literature. Hasegawa et al. (2008) and Piatti et al. (2010) obtained the color-magnitude diagram of 
Berkeley 26 in the BVI photometric system. Tadross (2008) analyzed near-infrared $J H K$ photometry obtained from $2 \mathrm{MASS}^{4}$. All of these authors derived the age, reddening, distance, and metallicity by means of isochrone fitting. There are some discrepancies between the values obtained by each of them. There is good agreement, within the uncertainties, in the values derived by Hasegawa et al. (2008) and Piatti et al. (2010), but not between them and the Tadross (2008) measurements. For example, Hasegawa et al. (2008) and Piatti et al. (2010) derived an age of $4.5 \mathrm{Gyr}$ and $4 \mathrm{Gyr}$, respectively. In contrast, Tadross (2008) obtained a much younger age of 0.6 Gyr. A reddening of $E(B-V)=0.61$ was derived by Hasegawa et al. (2008), which is between the values derived by Tadross (2008) of $E(B-V)=0.5$, and Piatti et al. (2010) of $E(B-V)=0.75$. In the case of the distance modulus, the obtained values range from $(m-M)_{0}=12.6$ (Tadross 2008) to 14.45 (Hasegawa et al. 2008). Piatti et al. (2010) derived a value between both of $(m-M)_{0}=13.17$. As was noted by Piatti et al. (2010), the poor quality of the data used by Tadross (2008), mainly owing to the techniques that they used to decontaminate the foreground contribution and select the cluster members, may explain the discrepancies with other investigations in the literature. For this reason, we used in our work the distance modulus and reddening obtained by averaging the values derived by Hasegawa et al. (2008) and Piatti et al. (2010) listed in Table 2.

A total of six stars were observed along the Berkeley 26 line of sight. The spectrum of the star 1650 contains clear molecular bands. Moreover, this star has a larger radial velocity than the other observed stars. For these reasons, we use this star in neither the radial velocity nor the metallicity determinations. The star 1038 is not a RGB and was used only for the radial velocity determination. Therefore, excluding star 1650, we derived a mean radial velocity for this cluster of $\left\langle V_{r}\right\rangle=68 \pm 12 \mathrm{~km} \mathrm{~s}^{-1}$.

Only Piatti et al. (2010) provided an estimation of the Berkeley 26 metallicity of $[\mathrm{Fe} / \mathrm{H}]=-0.70$ dex. Hasegawa et al. (2008) assumed a metallicity of $[\mathrm{Fe} / \mathrm{H}] \sim-0.35$ for this cluster. We derived $[\mathrm{Fe} / \mathrm{H}]=-0.43 \pm 0.20$ and $-0.35 \pm 0.17$ dex using $M_{V}$ and $M_{I}$, respectively. These values are in relatively good agreement with the photometric estimations taken into account the uncertainties. As explained above, Tadross (2008) derived a different distance modulus and reddening for Berkeley 26 than those used here. When these values were used in the metallicity determination, we obtained $[\mathrm{Fe} / \mathrm{H}]=+0.09$ and +0.06 in the $V$ and $I$ bandpasses, respectively.

\subsection{Berkeley 70}

Berkeley 70 is a rich OC, which probably belongs to the Perseus arm (Carraro et al. 2008). It shows a highly populated mainsequence and a well-developed RGB. A significant amount of blue stranglers can also be observed. To our knowledge, only two studies have determined the properties of this cluster, using both $U B V$ photometry (Ann et al. 2002; Hasegawa et al. 2004). The reddenings and distances determined by them are the same within the uncertainties. There is a slight difference in the ages obtained, although they are consistent with the uncertainties. While Ann et al. (2002) determined an age of 4 Gyr, Hasegawa et al. (2004) obtained 2.8 Gyr.

\footnotetext{
4 2MASS (Two Micron All Sky Survey) is a joint project of the University of Massachusetts and the Infrared Processing and Analysis Center/California Institute of Technology, funded by the National Aeronautics and Space Administration and the National Science Foundation.
}

The eight observed stars have similar radial velocities so we conclude that all of them are members of Berkeley 70. They define a narrow velocity distribution (top-right panel of Fig. 2). We derive a mean radial velocity for this clusters of $\left\langle V_{r}\right\rangle=$ $-15 \pm 7 \mathrm{~km} \mathrm{~s}^{-1}$.

For Berkeley 70 , we derived a metallicity of $[\mathrm{Fe} / \mathrm{H}]=$ $-0.02 \pm 0.18$ and $-0.01 \pm 0.14$ dex using $V$ and $I$ magnitudes, respectively. To our knowledge, the only determinations of metallicity for this cluster available in the literature come from isochrone fitting, and they are all lower than obtained here. Ann et al. (2002) determined a metallicity of -0.32 dex, while Hasegawa et al. (2004) obtained a slightly more metal-poor value of -0.48 dex. This discrepancy may be explained by the large uncertainties involved in the metallicity estimation from isochrone fitting owing to the limitation of the method itself and to the sparse color magnitude-diagram used as a reference.

\subsection{NGC 1798}

NGC 1798, also denoted as Berkeley 16, is another poorly studied OC located in the Auriga constellation. Its color-magnitude diagram shows a well-populated main-sequence and red clump, and a poorly defined RGB. The reddening towards the NGC 1798 is relatively high, $E(B-V)=0.51 \pm 0.04$ (Park \& Lee 1999; Dutra \& Bica 2000; Tadross et al. 2002; Salaris et al. 2004; Maciejewski \& Niedzielski 2007), which is in part explained by its proximity to the Galactic plane. An age of $1.4 \pm 0.2 \mathrm{Gyr}$ has been estimated by most of the previous investigations in this cluster (e.g. Janes \& Phelps 1994; Park \& Lee 1999; Lata et al. 2002; Salaris et al. 2004; Maciejewski \& Niedzielski 2007). These works have also obtained a similar distance modulus for this cluster of $(m-M)_{0}=13.1 \pm 0.2$.

The six stars observed in the NGC 1798 field seem to be members of this cluster based on their radial velocity. The mainsequence star 608 was observed because it was aligned with the slit in the observations of star 13 but it was only used for the radial velocity analysis. We determined a mean radial velocity of $\left\langle V_{r}\right\rangle=2 \pm 10 \mathrm{~km} \mathrm{~s}^{-1}$ for this cluster.

For the five RGB stars observed in NGC 1798, we inferred metallicities of $[\mathrm{Fe} / \mathrm{H}]=-0.16 \pm 0.10$ and $-0.12 \pm 0.07 \mathrm{dex}$ using $V$ and $I$ magnitudes, respectively. There are a handful of metallicity estimations for NGC 1798 in the literature. All of them assign a metallicity lower than that obtained here, $[\mathrm{Fe} / \mathrm{H}]=-0.46 \pm 0.10$ dex (Park \& Lee 1999; Tadross 2001, 2003; Paunzen et al. 2010). As in the case of Berkeley 70, this disagreement may be explained by the large uncertainties in the metallicities derived from isochrone fitting.

\subsection{NGC 2266}

NGC 2266, also known as Melotte 50, is a rich and wellcondensed system. It is the youngest cluster of our sample with an age similar to that of the Hyades, $(0.7 \pm 0.1 \mathrm{Gyr}$; Kaluzny \& Mazur 1991; Salaris et al. 2004; Monteiro et al. 2010; Dias et al. 2012), although some authors have obtained a slightly older age (1.2 \pm 0.1 Gyr; Maciejewski \& Niedzielski 2007; Maciejewski et al. 2008). Its color-magnitude diagram has a populated mainsequence, especially in the turn-off area, and a well-defined redclump located at $V \sim 13.7$ with a few stars above it (Fig. 1). The contamination of the color-magnitude diagram seems to be almost negligible. We used the reddening and distance derived by Dias et al. (2012, see Table 2), which are similar, within 
the uncertainties to others available in the literature (e.g. Lata et al. 2002; Paunzen \& Netopil 2006; Maciejewski et al. 2008; Monteiro et al. 2010).

Six stars were observed in this cluster. However, it seems that only four of them, including the main-sequence object 75 , are true members of the cluster. The other two, stars 34 and 67, have a radial velocity differing from the average of the other stars much more than the expected uncertainties, hence may not be members of this cluster. Excluding these two stars, we obtained a mean radial velocity of $\left\langle V_{r}\right\rangle=-16 \pm 15 \mathrm{~km} \mathrm{~s}^{-1}$ for NGC 2266.

Since one of the four stars confirmed as a NGC 2266 member is a main-sequence object, its metallicity determination is based on only three stars. Moreover, I magnitudes are unavailable for these stars. We therefore, obtained a metallicity of $[\mathrm{Fe} / \mathrm{H}]=$ $-0.38 \pm 0.07$ dex derived only from $V$ magnitudes. This value is in very good agreement with the following estimates available in the literature: $-0.26 \pm 0.20$ dex (Paunzen et al. 2010); -0.68 (Maciejewski et al. 2008); $-0.26 \pm-0.18$ (Kaluzny \& Mazur 1991); and -0.35 (Monteiro et al. 2010; Dias et al. 2012). We note, however, that Salaris et al. (2004) assigned a more metalrich metallicity of $0.00 \pm 0.20$ dex.

\section{Comparison with trends in the Galactic disk}

As mentioned earlier, OCs are fundamental test particles in the study of the formation and evolution of the Galactic disk. In particular, they are used to trace the existence of trends in metallicity with Galactocentric distance, Rgc, the vertical distance to the Galactic plane, $z$, and age, providing fundamental constraints on chemical evolution models. All the investigations based on OCs agree that the iron content decreases almost linearly with increasing radius (e.g. Friel et al. 2002; Pancino et al. 2010). However, investigations based on samples containing clusters at larger distances found that the metallicity decreases as a function of increasing radius to $R_{\mathrm{gc}} \sim 12.5 \mathrm{kpc}$ and appears to flatten from there outwards (e.g. Yong et al. 2005; Sestito et al. 2008; Andreuzzi et al. 2011; Carrera \& Pancino 2011). Moreover, it seems that this gradient has evolved with time, having been steeper in the past (e.g. Magrini et al. 2009; Andreuzzi et al. 2011; Carrera \& Pancino 2011). However, there are no hints of the existence of an age-metallicity relationship (e.g. Friel 1995). This indicates that the chemical enrichment of OCs is modulated by their location in the Galaxy and not by the moment at which they formed (Carrera \& Pancino 2011).

It is helpful to check how the clusters studied in this paper fit the general trends observed in the Galactic disk. This comparison, of course, should be interpreted with caution because the results obtained here should be confirmed by analyses based on high-resolution spectra. In all cases, this comparison is interesting because of the location of these clusters at relatively large distances from the Galactic anticenter $\left(R_{\mathrm{gc}}<10 \mathrm{kpc}\right)$. To perform this comparison, we used the OC compilation obtained by Carrera \& Pancino (2011), which contains data for 89 clusters whose chemical abundances were derived by investigations based on high-resolution spectra $(R \geq 20000)$ available in the literature. We refer the reader to this paper for details. The run of metallicity with $R_{\mathrm{gc}}$, age, and $|z|$ for this sample have been plotted in the top, middle, and bottom panels of Fig. 5, respectively. The solid lines in the top panel are the linear fits to the sample assuming a change in the slope at $R_{\mathrm{gc}} \sim 12.5 \mathrm{kpc}$, while the long-dashed line is the linear fit to the whole sample. Clusters in the inner $10 \mathrm{kpc}$ and from there outwards have been plotted with dark and light gray circles, respectively, in the three panels.
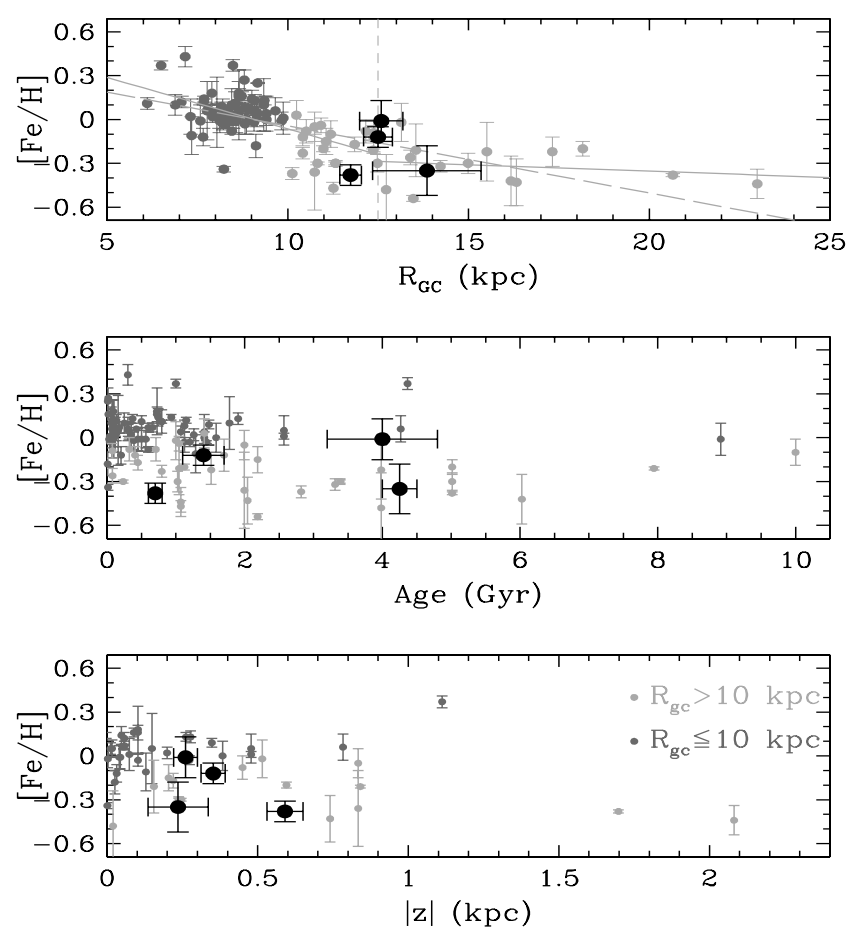

Fig. 5. Run of $[\mathrm{Fe} / \mathrm{H}]$ with $R_{\mathrm{gc}}$ (top), age (middle), and $|z|$ (bottom) of the OCs in the Carrera \& Pancino (2011) compilation. Dark and light gray points are clusters in the inner $10 \mathrm{kpc}$ and from there off, respectively. Black points are the clusters studied in this paper.

The four clusters studied here have been overplotted with black symbols. Berkeley 26, in spite of the uncertainty in its distance, and NGC 1798 closely follow the general trends observed in other coeval clusters located at similar Galactocentric distances. NGC 2266 seems to be slightly younger than other metal-poor systems of similar metallicity situated at the same distance. Nevertheless, its metal content is akin to other OCs located away from the Galactic plane, such as NGC 2266. In all cases, this comparison must be considered with caution because its metallicity has been obtained from only three stars. In contrast, Berkeley 70, which has a more robust metallicity determination based on eight stars, appears more metal-rich than other systems located at $\sim 12.5 \mathrm{kpc}$. There are a few clusters with similar ages and metallicities to Berkeley 70 , but all of them are situated in the inner $10 \mathrm{kpc}$. Although it is beyond the scope of this paper, one explanation could be that this cluster was formed at a smaller Galactocentric distance and then migrated to its current location. However, more information than is currently available is needed to confirm or disprove this hypothesis.

\section{Conclusions}

We have analyzed medium-resolution spectra $(R \sim 8000)$ in the infrared CaT region ( $~ 8500 \AA)$ of several stars in four OCs: Berkeley 26, Berkeley 70, NGC 1798, and NGC 2266. To our knowledge, this is the first time that these clusters have been studied spectroscopically. Our main results can be summarised as follows:

- For Berkeley 26, we derived a mean radial velocity of $\left\langle V_{r}\right\rangle=$ $68 \pm 12 \mathrm{~km} \mathrm{~s}^{-1}$ based on six stars. One of them is a mainsequence star that was not used in the metallicity determinations. Using $V$ and $I$ magnitudes, we determined metallicities of $[\mathrm{Fe} / \mathrm{H}]=-0.43 \pm 0.20$ and $-0.35 \pm 0.17$ dex, respectively. 
- The eight observed stars in Berkeley 70 were confirmed as members based on their radial velocity. For them, we derived a mean radial velocity of $\left\langle V_{r}\right\rangle=-15 \pm 7 \mathrm{~km} \mathrm{~s}^{-1}$ and metallicities of $[\mathrm{Fe} / \mathrm{H}]=-0.02 \pm 0.18$ and $-0.01 \pm 0.14$ dex based on $V$ and $I$ magnitude data, respectively.

- We derived a mean radial velocity for NGC 1798 of $\left\langle V_{r}\right\rangle=$ $2 \pm 10 \mathrm{~km} \mathrm{~s}^{-1}$ from six stars, although one object is on the main sequence. For the RGB stars, we obtained a metallicity of $[\mathrm{Fe} / \mathrm{H}]=-0.16 \pm 0.10$ and $-0.12 \pm 0.07 \operatorname{dex}$ in $V$ and $I$ bandpasses, respectively.

- In the case of NGC 2266, its mean radial velocity, $\left\langle V_{r}\right\rangle=$ $-16 \pm 15 \mathrm{~km} \mathrm{~s}^{-1}$ was obtained for four objects. A metallicity of $[\mathrm{Fe} / \mathrm{H}]=-0.38 \pm 0.07$ dex was derived from the $V$ magnitudes of the three RGB stars observed in this cluster.

Finally, we investigated how the four analyzed clusters fit the trends defined by other well-studied OCs. This comparison is motivated by our clusters being situated at distances where other investigations have observed a change in the slope of the metallicity gradient. In general, Berkeley 26 and NGC 1798 follow the trends described by other coeval systems situated at the same distance. In the case of NGC 2266, its height above the Galactic plane can explain its low metallicity compared to other clusters of the same age. In contrast, Berkeley 70 seems to be more metal-rich than other coeval clusters situated at similar distances. We suggest that this cluster may have formed at relatively small Galactocentric distances and has migrated outwards with time. Nevertheless, more information is needed to confirm or discard this hypothesis.

Acknowledgements. R.C. acknowledge the support of the Spanish Ministry of Economy and Competitiveness (Plan Nacional de Investigación Científica, Desarrollo, e Investigación Tecnológica, AYA2010-16717). R.C. also acknowledges the funds of the Spanish Ministry of Science and Innovation under the Juan de la Cierva fellowship. This research has made use of the WEBDA database, operated at the Institute for Astronomy of the University of Vienna, and the SIMBAD database, operated at CDS, Strasbourg, France

\section{References}

Andreuzzi, G., Bragaglia, A., Tosi, M., \& Marconi, G. 2011, MNRAS, 412, 1265 Ann, H. B., Lee, S. H., Sung, H., et al. 2002, AJ, 123, 905

Armandroff, T. E., \& Da Costa, G. S. 1991, AJ, 101, 1329

Armandroff, T. E., \& Zinn, R. 1988, AJ, 96, 92

Bartašiūtè, S., Janusz, R., Boyle, R. P., \& Philip, A. G. D. 2011, Baltic Astron., 20,27

Carrera, R., \& Pancino, E. 2011, A\&A, 535, A30

Carrera, R., Gallart, C., Pancino, E., \& Zinn, R. 2007, AJ, 134, 1298
Carrera, R., Gallart, C., Aparicio, A., \& Hardy, E. 2011, AJ, 142, 61 Carraro, G., Vallenari, A., Girardi, L., \& Richichi, A. 1999, A\&A, 343, 825

Carraro, G., Vázquez, R. A., \& Moitinho, A. 2008, A\&A, 482, 777

Carretta, E., \& Gratton, R. G. 1997, A\&AS, 121, 95

Carretta, E., Bragaglia, A., Gratton, R., D’Orazi, V., \& Lucatello, S. 2009, A\&A, 508,695

Cenarro, A. J., Cardiel, N., Gorgas, J., et al. 2001, MNRAS, 326, 959

Chen, L., Hou, J. L., \& Wang, J. J. 2003, AJ, 125, 1397

Clariá, J. J., Piatti, A. E., \& Lapasset, E. 1998, A\&AS, 128, 131

Clariá, J. J., Mermilliod, J.-C., \& Piatti, A. E. 1999, A\&AS, 134, 301

Cole, A. A., Smecker-Hane, T. A., Tolstoy, E., Bosler, T. L., \& Gallagher, J. S. 2004, MNRAS, 347, 367

Dias, W. S., Alessi, B. S., Moitinho, A., \& Lépine, J. R. D. 2002, A\&A, 389, 871

Dias, W. S., Monteiro, H., Caetano, T. C., \& Oliveira, A. F. 2012, A\&A, 539, A125

Dutra, C. M., \& Bica, E. 2000, A\&A, 359, 347

Friel, E. D. 1995, ARA\&A, 33, 381

Friel, E. D., \& Janes, K. A. 1993, A\&A, 267, 75

Friel, E. D., Janes, K. A., Tavarez, M., et al. 2002, AJ, 124, 2693

Hasegawa, T., Malasan, H. L., Kawakita, H., et al. 2004, PASJ, 56, 295

Hasegawa, T., Sakamoto, T., \& Malasan, H. L. 2008, PASJ, 60, 1267

Janes, K. A. 1977, AJ, 82, 35

Janes, K. A. 1979, ApJS, 39, 135

Janes, K. A., \& Phelps, R. L. 1994, AJ, 108, 1773

Kaluzny, J., \& Mazur, B. 1991, Acta Astron., 41, 191

Lata, S., Pandey, A. K., Sagar, R., \& Mohan, V. 2002, A\&A, 388, 158

Maciejewski, G., \& Niedzielski, A. 2007, A\&A, 467, 1065

Maciejewski, G., Boeva, S., Georgiev, T., et al. 2008, Baltic Astron., 17, 51

Magrini, L., Sestito, P., Randich, S., \& Galli, D. 2009, A\&A, 494, 95

Mermilliod, J.-C. 1995, Information \& On-Line Data in Astronomy, 203, 127

Monteiro, H., Dias, W. S., \& Caetano, T. C. 2010, A\&A, 516, A2

Panagia, N., \& Tosi, M. 1980, A\&A, 81, 375

Pancino, E., Carrera, R., Rossetti, E., \& Gallart, C. 2010, A\&A, 511, A56

Parisi, M. C., Clariá, J. J., Piatti, A. E., \& Geisler, D. 2005, MNRAS, 363, 1247

Park, H. S., \& Lee, M. G. 1999, MNRAS, 304, 883

Paunzen, E., \& Netopil, M. 2006, MNRAS, 371, 1641

Paunzen, E., Maitzen, H. M., Rakos, K. D., \& Schombert, J. 2003, A\&A, 403, 937

Paunzen, E., Heiter, U., Netopil, M., \& Soubiran, C. 2010, A\&A, 517, A32

Piatti, A. E., Clariá, J. J., \& Ahumada, A. V. 2010, MNRAS, 402, 2720

Pont, F., Zinn, R., Gallart, C., Hardy, E., \& Winnick, R. 2004, AJ, 127, 840

Rutledge, G. A., Hesser, J. E., \& Stetson, P. B. 1997, PASP, 109, 907

Salaris, M., Weiss, A., \& Percival, S. M. 2004, A\&A, 414, 163

Sestito, P., Bragaglia, A., Randich, S., et al. 2008, A\&A, 488, 943

Starkenburg, E., Hill, V., Tolstoy, E., et al. 2010, A\&A, 513, A34

Tadross, A. L. 2001, New A, 6, 293

Tadross, A. L. 2003, New A, 8, 737

Tadross, A. L. 2008, MNRAS, 389, 285

Tadross, A. L., Werner, P., Osman, A., \& Marie, M. 2002, New A, 7, 553

Twarog, B. A., Ashman, K. M., \& Anthony-Twarog, B. J. 1997, AJ, 114, 2556

Vallenari, A., Carraro, G., \& Richichi, A. 2000, A\&A, 353, 147

Warren, S. R., \& Cole, A. A. 2009, MNRAS, 393, 272

Yong, D., Carney, B. W., \& Teixera de Almeida, M. L. 2005, AJ, 130, 597

Zdanavičius, J., Vrba, F. J., Zdanavičius, K., Straižys, V., \& Boyle, R. P. 2011, Baltic Astron., 20, 1 